\title{
BRUNO BAUER - EIN MITSTREITER IM RINGEN UM DIE KOOPERATION E-MEDIEN ÖSTERREICH
}

\section{von Helmut Hartmann ${ }^{1}$}

Als um die Jahrtausendwende weltweit Konsortien wissenschaftlicher Bibliotheken zur Nutzung von E-Zeitschriften gegründet wurden, herrschte in Österreich in der Administration und Budgetierung von Universitätsbibliotheken immer noch die traditionelle Kameralistik mit ihren von jeder einzelnen Bibliothek in Jahresfrist zu verbrauchenden Budgets ohne jede Möglichkeit bibliotheksübergreifender Zusammenschlüsse. Nach Fortbildungsaufenthalten in den USA, England und Deutschland und Vertiefung in die Vor- und Nachteile verschiedener Konsortienmodelle bemühte sich der Verfasser, Mitstreiter für die Einführung dieser Bezugsform auch in Österreich zu finden. ${ }^{2}$

Kommunikation und Koordination zu ermöglichen bildete damals die Voraussetzung für die Etablierung sinnvoller Konsortialstrukturen in Österreich - und genau dafür stellte sich Bruno Bauer zur Verfügung: Einerseits rührte er unermüdlich die Werbetrommel inner- und außerhalb der VÖB, andererseits konnte dank seiner Befürwortung der Vortragsraum der Zentralbibliothek für Medizin (wie die Bibliothek der Medizinischen Universität Wien vor deren Gründung 2004 noch hieß) für etliche Informationstreffen interessierter TeilnehmerInnen und Verhandlungen mit Großverlagen genützt werden. Nicht zuletzt dank Bruno Bauers Interesse gelang es bereits damals, sogar erste Nachschlagewerke durch Konsortialverträge online anzubieten: 2002 das klinische Wörterbuch Pschyrembel und ein Jahr später 8 englischsprachige medizinische Lehrbücher über die OVID-Plattform.

Dass es bei diesen oft Stunden dauernden und sehr arbeitsintensiven Meetings ohne Tageslicht und mit nur von der Klimaanlage eingeblasener Frischluft nicht zur alsbaldigen Erschöpfung der meisten Anwesenden kam, war seiner vorbildlichen Gastfreundschaft zu verdanken. Immer wurden reichlich Getränke vorbereitet, und in der Mittagspause sorgten von guten Geistern herangebrachte Servierwagen mit belegten Weckerln und köstlichen Mehlspeisen für eine entspannte Atmosphäre, als deren Folge in der „zweiten Halbzeit" so mancher Interessensausgleich erfolgte, so manche Differenz beigelegt werden konnte und so mancher Vertrag ausgehandelt wurde. 
Wie wichtig Bruno Bauer diese Veranstaltungen waren, bewies sein ungebrochener Einsatz für den Erfolg des am 1. Juli 2005 von 13 österreichischen Universitätsbibliotheken gegründeten Konsortiums für E-Medien, der „Kooperation E-Medien Österreich“, auch nach seiner Ernennung zum Leiter der Bibliothek in der Nachfolge von HR Dr. Helmut Leitner im November 2005. Wann immer es seine Zeit gestattete, nahm er an KEMÖ-Treffen teil, und umgekehrt wurde der Verfasser als Leiter der Geschäftsstelle der KEMÖ von Anfang an zu den von Bruno Bauer im Mai 2005 eingeführten halbjährlichen Treffen der österreichischen Medizinbibliothekarinnen und -bibliothekare eingeladen, um so gerade diese Nutzergruppe gezielt in die neue Struktur einzubinden, da in den Fachgebieten Medizin und Naturwissenschaften die Entwicklung hin zu E-Zeitschriften am rasantesten vor sich ging.

Doch nicht nur der persönliche Erfahrungs- und Informationsaustausch bedeutete Bruno Bauer viel, seine schier grenzenlose Begeisterung für wissenschaftliches Publizieren, sein Eifer, seine Freude an der Analyse mit der Einführung der E-Medien verbundener Phänomene erwies sich als so „ansteckend“, dass der Verfasser gern der Einladung des Verewigten nachkam, selbst für verschiedene bibliothekswissenschaftliche Zeitschriften Beiträge zu erstellen - darunter natürlich die unter der Chefredaktion Bruno Bauers von der Arbeitsgemeinschaft für Medizinisches Bibliothekswesen e.V. (AGMB) herausgegebene Zeitschrift medizin - bibliothek - information. ${ }^{3}$ Darüber hinaus gehört es bis heute zu den prägenden Erfahrungen des Verfassers, an der von Bruno Bauer angestoßenen gemeinsamen Online-Benutzerbefragung an zehn österreichischen Universitäten $2083^{4}$ mitgewirkt zu haben, oder parallel zu seiner Untersuchung der Finanzierbarkeit von Open Access an der Bibliothek der Medizinischen Universität Wien ${ }^{5}$ diese Option für den Zeitschriftenbestand der Universität Graz ausgelotet zu haben. ${ }^{6}$

Die zuletzt genannten drei Veröffentlichungen sind nicht zufällig Tagungsbeiträge. Ein begnadeter Netzwerker wie Bruno Bauer durfte auf nationalen und internationalen Bibliothekstagungen, Konferenzen und Fortbildungsveranstaltungen einfach nicht fehlen - er war mit seinem umfassenden Wissen, seinen minutiösen Recherchen, seinem Gespür für verborgene Zusammenhänge ein Gewinn für jede Veranstaltung. Gerne war er bereit, sich mit stets erstklassig vorbereiteten Beiträgen einzubringen, wenn man als Veranstalter einen call for papers aussendete; umgekehrt bat er charmant und unwiderstehlich um Beiträge, wenn er einem Programmkomitee angehörte. Ihm und 
der leider 2017 ebenfalls viel zu früh von uns gegangenen Kollegin $\mathrm{Dr}^{\text {in }}$ Eveline Pipp ist es neben etlichen sich mittlerweile bereits im Ruhestand befindlichen BibliotheksdirektorInnen ${ }^{7}$ und KollegInnen zu danken, dass die VÖB mit ihren Kongressen, Fortbildungsveranstaltungen, Kommissionen und Arbeitskreisen die Entwicklung des österreichischen Konsortienwesens im Allgemeinen und der KEMÖ im Besonderen mitgetragen hat.

Am Schluss dieser Würdigung bleibt dem Verfasser, der nun auch schon einige Jahre in Pension ist, nur noch, ein Wort des persönlichen Dankes zu sagen für mehr als zwei Jahrzehnte des immer interessanten Meinungsaustauschs, des hilfsbereiten Eingehens auf Vorschläge, aber auch des wohl überlegten Widerspruchs. Man wird sich auf keinem Bibliothekartag mehr begegnen, bei keinem VÖB-Stammtisch mehr treffen, das letzte Bier wird ungetrunken bleiben, aber Brunos offenes, menschenfreundliches Lächeln wird uns begleiten - Trost und Ansporn ein Leben lang.

Helmut Hartmann E-Mail: helmut.hartmann@drei.at

DOI: https://doi.org/10.31263/voebm.v74i1.6086

(C) Helmut Hartmann

Dieses Werk ist - exkl. einzelner Logos und Abbildungen - lizenziert unter einer Creative-Commons-Lizenz Namensnennung 4.8 International-Lizenz 
1 Erster Leiter der Geschäftsstelle der auf seine Initiative hin gegründeten Kooperation E-Medien Österreich.

2 Eine ausführliche Darstellung der Entwicklung der KEMÖ bietet: Bauer, Bruno u. Hartmann, Helmut: Bibliothekskonsortien in Österreich: Helmut Hartmann [...] beantwortet 11 Fragen von Bruno Bauer. In: Mitteilungen der VÖB 66 (2013), H. 3/4, S. 422-447. Die gesamte Ausgabe ist online verfügbar unter: https://phaidra.univie. ac.at/o:309283

3 Hartmann, Helmut: E-Bücher. Vom Project Gutenberg zum Paper-Like Display Book. In: medizin-bibliothek-information 3 (2003), H. 3, S. 12-15. - Weitere hier angesprochene Veröffentlichungen werden in der Bibliographie des unter Anm. 2 zitierten Beitrags aufgelistet.

4 Bauer, Bruno u. a.: Wie beurteilen Nutzer unser elektronisches Medien- und Dienstleistungsangebot? Ausgewählte Ergebnisse der gemeinsamen Online-Benutzerbefragung 2003 an zehn österreichischen Universitäts- und Zentralbibliotheken. In: Enichlmayr, Christian (Hrsg.): Bibliotheken - Fundamente der Bildung (28. Österreichischer Bibliothekartag 2004). Weitra: Bibliothek der Provinz 2005, S. 151-189.

5 Bauer, Bruno: Open Access Publishing - Trends in Deutschland, Österreich und der Schweiz. Initiativen, Projekte, Stellenwert. In: Eveline Pipp (Hrsg.): Zugang zum Fachwissen. ODOK`Q5 ; 11. Österreichisches Online-Informationstreffen, 12. Österreichischer Dokumentartag ; 13.-16. September 2005, Freie Universität Bozen (Schriften der Vereinigung Österreichischer Bibliothekarinnen und Bibliothekare 1) Graz-Feldkirch: Neugebauer 2007, S. 194-220.

6 Hartmann, Helmut: Open Access - Bibliotheken unterwegs ins Gelobte Land? In: Eveline Pipp (Hrsg.): Zugang zum Fachwissen. ODOK` $85 ; 11$. Österreichisches Online-Informationstreffen, 12. Österreichischer Dokumentartag ; 13.-16. September 2005, Freie Universität Bozen (Schriften der Vereinigung Österreichischer Bibliothekarinnen und Bibliothekare 1). Graz-Feldkirch: Neugebauer 2007, S. 147-159.

7 Namentlich erwähnt sei hier HR Dr. Heinz Hauffe von der UB Innsbruck, der „Urvater“ der E-Zeitschriften an österreichischen Universitätsbibliotheken. Für seine großzügige Unterstützung bei der Einführung von E-Zeitschriften ist inm der Verfasser noch heute dankbar. 